\title{
Sacubitril/Valsartan in Management of Heart Failure: A Review of Safety, Efficacy and Cost-Effectiveness
}

\section{Samer Ellahham*}

Cleveland Clinic Abu Dhabi and Cleveland Clinic Foundation, Abu Dhabi, United Arab Emirates

*Corresponding author: Dr. Samer Ellahham, Cleveland Clinic Abu Dhabi and Cleveland Clinic Foundation, UAE/CCF 1950 Richmond Road, TR303, Lyndhurst, OH 44124, Abu Dhabi, UAE, Tel: 971508113142; E-mail: ellahas@clevelandclinicabudhabi.ae

Received date: November 20, 2018; Accepted date: December 24, 2018; Published date: December 31, 2018

Copyright: ( 2018 Ellahham S. This is an open-access article distributed under the terms of the Creative Commons Attribution License, which permits unrestricted use, distribution, and reproduction in any medium, provided the original author and source are credited.

\begin{abstract}
Heart failure (HF) is a leading cause of mortality and morbidity across the globe. High prevalence, frequent hospitalization, longterm healthcare, absenteeism from work, and death upsurge the economic burden of the HF. For almost two decades, the angiotensin converting enzyme inhibitors (ACEIs), angiotensin II receptor blockers (ARBs), and beta-blockers (BBs) have remained the standard care of management for HF. But despite being efficacious and cost-effective theses standard treatment were unable reduce events of rehospitalization and improve quality of life for patients with HF. Recent innovative discovery of angiotensin receptor/neprilysin inhibitor (ARNI) therapy changed the entire outlook for management of HF. Sacubitril/Valsartan an ARNI proved out to be a revolutionary drug for patients of HF. Compared to other drug classes, Sacubitril/Valsartan combination therapy is more efficacious, have lesser adverse effect, and improves overall quality of life of HF patients with reduced ejection fraction. However, the cost-effectiveness of Sacubitril/Valsartan remained questionable in developing parts of world. At the current pricing Sacubitril/Valsartan was found to be cost-effective only at specific threshold of willingness to pay of the patients. Better Federal healthcare policies, new-pricing strategies, robust long-term real-world estimates of this drug are required to improve the cost-effective profile of this drug.
\end{abstract}

Keywords: Heart failure; Sacubitril/Valsartan; LCZ 696; costeffectiveness

\section{Introduction}

Heart failure (HF) is a leading cause of mortality and morbidity with an estimated prevalence of 26 million worldwide [1]. Ischemic heart disease, hypertension, and diabetes are the three most common risk factors that increase the risk of HF [2]. Other less common factors attributable to HF are; cardiomyopathies, infections (e.g., viral myocarditis, Chagas' disease), toxins (e.g., alcohol, cytotoxic drugs), valvular disease, and prolonged arrhythmias [2].

Clinically, cardiac dysfunction caused by HF is classified into systolic HF and diastolic HF [3]. Systolic HF or HF with reduced ejection fraction (HFrEF) is due to depressed myocardial contractility therefore, resulting in left ventricular ejection fraction (LVEF) $\leq 40 \%$. Whereas, diastolic HF or HF with preserved ejection fraction (HFpEF) is due to reduced ventricular relaxation and filling hence, have LVEF $\geq$ $50 \%$ [3].

Data from the global registry of Hospitalized heart failure reports that the median length of stay in patients with heart failure ranges from 4 to 20 days and in-hospital mortality from $4 \%$ to $30 \%$ [1]. Recurrent hospital admissions, loss of work and productivity, and death are associated with a significant socioeconomic burden in patients with HF, amounting to an estimated cost of $\$ 108$ billion worldwide in 2012 [4]. Regional data from the INTERnational Congestive Heart Failure (INTER-CHF) study showed that socioeconomic burden of HF is considerably higher in Africa, Asia, the Middle East and South America region. Early age of onset of HF (average age 56.4 years) and large proportion of patients lacking health and medication insurance further adds to cost of healthcare in this region [4]. Hence, in today's growing armamentarium of HF therapies the cost-effectiveness of a drug plays a pivotal role while prescribing HF therapy, particularly in developing regions of the world.

\section{Objectives and Methods}

Sacubitril/Valsartan (SAC/VAL), is one of the newest additions in HF therapy. This review explores the recommended guidelines on combination therapy with SAC/VAL, advantages and disadvantages of this combination over traditional therapies, and effectiveness and safety of SAC/VAL in patients with HFrEF. Additionally, this review also scrutinizes the cost-effectiveness of SAC/VAL to understand the clinical utility this drug, specifically in developing parts of the world.

A search of PubMed (till June 2018) for studies evaluating the combination of SAC/VAL and long-term outcomes for the treatment of HF was conducted. Pre-defined MeSH terms "Sacubitrip" "Valsartan", "heart failure", and " $L C Z 696$ " were used along with Boolean operator "AND” for an extensive database search. Also, filters like 'English Language' and 'studies published in the last 5 years' were applied for a precise search. All the resulting 248 titles and abstracts were scanned for relevance. A total of twenty-six clinical trials were considered in the current review. Additionally, a manual search was conducted to include three clinical practice guidelines.

\section{Pathophysiology of Heart Failure}

Heart failure is a complex clinical syndrome resulting from various pathophysiological dysfunctions [2]. Normal cardiac functioning is regulated by a balanced relationship between myocardial contractility and the volume of blood circulated throughout the body [3]. In HF due to systolic dysfunction, there is a depressed myocardial contractility which corresponds to reduced cardiac output (CO) [3]. 
Decreased CO further leads to a decrease in mean arterial pressure (MAP) and therefore decreased tissue perfusion [3].

There are several compensatory mechanisms in HF that maintain adequate tissue perfusion, MAP, and CO during left ventricular failure. Three important compensatory mechanism regulating progression of HF are; Frank-Starling mechanism, neurohormonal activation, and ventricular remodeling [3].

The Frank-Starling mechanism works during the early stages of HF. It is related to left ventricular end-diastolic volume (LVEDV) [3]. Consequently, to an increased LVEDV, the left ventricular enddiastolic pressure (LVEDP) increases [3]. To compensate the increased LVEDP, the myocardium stretches and hence maintains the normal CO. Eventually with the progression of HF, the myocardium gets exhausted and $\mathrm{CO}$ cannot be further maintained by this mechanism [3].

Poor tissue perfusion and progressive decrease in MAP triggers the sympathetic nervous system (SNS) causing release of catecholamines (norepinephrine and epinephrine). Activation of SNS increases the heart rate, contractility, and vasoconstriction (in peripheral vasculature) thereby, increasing the MAP. Activity of SNS is mediated by three receptors viz. $\beta 1, \beta 2$, and $\alpha 1[2,3]$. Prolonged activation of SNS and $\beta 1, \beta 2$, and $\alpha 1$ receptors cause myocardial toxicity resulting in decreased ejection fraction (EF), arrhythmias, and tachycardia. Additionally, activation of the $\beta 1$ and $\alpha 1$ receptors also activates the renin angiotensin aldosterone system (RAAS) which causes vasoconstriction, sodium retention, and thirst, thereby augmenting MAP $[2,3]$.

Under normal circumstances, the activity of SNS and RAAS is balanced by natriuretic peptide system (NPS) to maintain homeostasis in body. Overactivity of SNS and RAAS triggers NPS to produce atrial natriuretic peptide (ANP), brain natriuretic peptide (BNP), and c-type natriuretic peptide (CNP) $[2,3]$. These natriuretic peptides induce vasodilation, natriuresis, and diuresis. With advancement of $\mathrm{HF}$, the production of biologically active ANP and BNP decrease. Furthermore, there is an increase in degradation of natriuretic peptides by neprilysin, increase in receptor-mediated clearance of natriuretic peptides in circulation, and desensitization of natriuretic peptide receptors in target organs [2,3]. Eventually, NPS is no longer able to compensate for overactivity of RAAS and SNS. Thus, there is an increase in $\mathrm{CO}$, blood pressure, and blood volume resulting in congestive symptoms.

Chronic progressive hemodynamic stresses from neurohormonal activation further strains heart leading to alterations in the size, shape, structure, and function of the ventricle resulting in ventricular remodeling [3]. Because of these mechanisms a vicious cycle develops which eventually results in progressive ventricular dysfunction and death [3].

\section{Development of Sacubitril/Valsartan}

For years, the clinical management of HF was based on treatment of the underlying cardiac condition, co-existing disease and volume overload [5]. Conventionally angiotensin-converting enzyme inhibitors (ACEIs), angiotensin receptor blockers (ARBs), beta blockers (BB), and mineralocorticoid receptor antagonist (MRA) have remained choice of drug therapy for HF (Figure 1) [6-8]. Even with these drug therapies prognosis seems to plateau over time in patients with HF. Epidemiological evidences suggest that mean survival rates (with ongoing drug therapy) in chronic HF are 80-90\%, 50-60\%, and $30 \%$ at 1,5 , and 10 years respectively [9]. This urged the clinical researcher to contemplate other endogenous escape pathways that could be used for developing target therapies. Explicit attention was paid to the RAAS and SNS pathway and new therapies were developed that could target both RAAS and NPS without increasing risk of hemodynamic stress. A conjugation molecule including an angiotensin II receptor blocker (valsartan) plus a neprilysin inhibitor (sacubitril) was synthesized and called SAC/VAL. This new molecule was regarded as the parent compound of a new class of drugs, termed angiotensin receptor neprilysin inhibitors (ARNI) [3].

\section{ard \\ PHARMACOTHERAPY IN HEART FAILURE
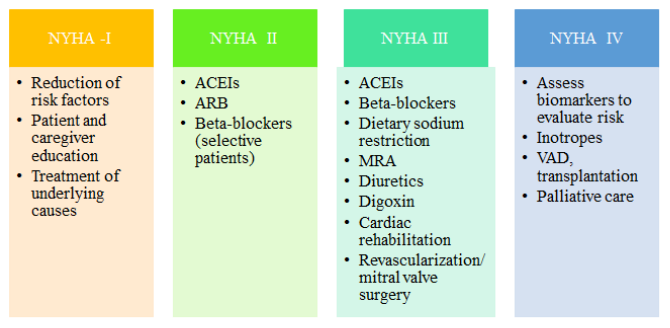 \\ Figure 1: Standard Pharmacotherapy for Heart Failure [6-8] (NYHA: New York Heart Association, ACEIs: angiotensin- converting enzyme inhibitors, ARB: angiotensin receptor blockers, MRA: mineralocorticoid receptor antagonist, VAD: ventricular assist device).}

\section{Mechanism of Action of Sacubitril/Valsartan}

Sacubitril/Valsartan is a 1:1 molar ratio combination of sacubitril (AHU377), a prodrug that is later metabolized to the neprilysin inhibitor LBQ657, and valsartan, an angiotensin II receptor blocker [10].

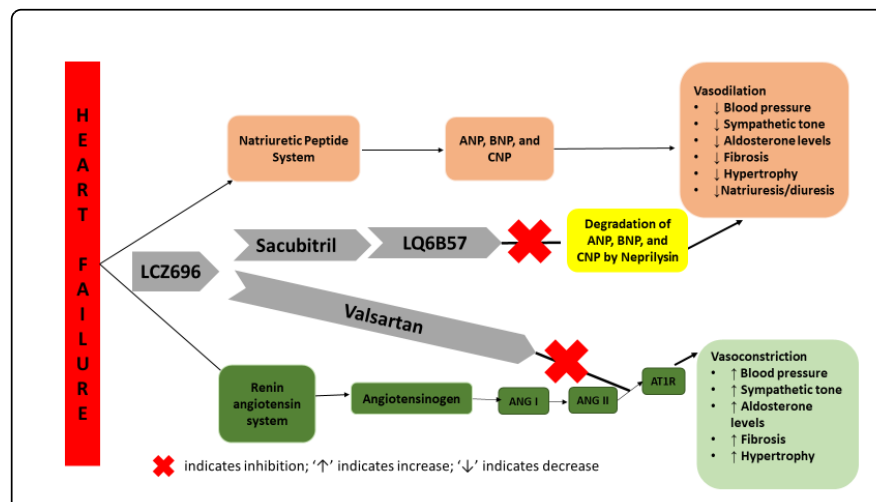

Figure 2: Mechanism of Action of Sacubitril/valsartan (ANP: Atrial natriuretic peptide, BNP: B-Type natriuretic peptide, CNP: C-Type natriuretic peptide, ANG I: Angiotensin I, ANG II: Angiotensin II, AT1R: Angiotensin 1 receptors). 
Once ingested, SAC/VAL undergoes rapid dissociation into valsartan and sacubitril. The sacubitril component augments NPS while valsartan inhibits the RAAS, thereby, improving the symptoms and inhibiting the progression of HF (Figure 2) [11]. Furthermore, SAC/VAL could also be helpful in post myocardial infarction remodeling [11]. An animal study used an in-silico systems biology approach to analyze myocardial infarction in swine to address postinfarction ventricular remodeling. Later, the swine genome hits were mapped to their human equivalents [11]. Results of this study showed that SAC/VAL reduces ventricular remodeling by decreasing rate of cardiomyocyte cell death, hypertrophy, and impaired myocyte contractility [11]. These findings provide the rationale for using SAC/VAL in patients with HF.

\section{The PARADIGM-HF Trial}

Prospective comparison of ARNI with ACEI to determine impact on global mortality and morbidity in heart failure (PARADIGM-HF) trial was performed to study the possible role of ARNI therapy in management of HF [12]. First-in-its-class ARNI therapy using SAC/VAL was compared with enalapril for its efficacy and safety in HF patients with a reduced ejection fraction [12]. This double-blinded study included 8442 patients with New York Heart Association (NYHA) class II, III, or IV heart failure and an ejection fraction of $40 \%$ or less. In addition to the recommended therapy, both test groups received either SAC/VAL (at a dose of $200 \mathrm{mg}$ twice daily) or enalapril (at a dose of $10 \mathrm{mg}$ twice daily) [12].

The primary endpoint was composite of death from cardiovascular causes or a first hospitalization for heart failure. However, the trial also studied a difference in the rates of death from cardiovascular causes.

The results of this study showed superiority of SAC/VAL over enalapril in reducing risk of death and first hospitalization due to worsening of HF [12]. Death from cardiovascular causes or hospitalization for HF was lower in SAC/VAL group [ $n=914,(21.8 \%)]$ than in the enalapril group $[\mathrm{n}=1117,(26.5 \%)]$, hazard ratio (HR) 0.80 , 95\% confidence interval (CI), 0.73 to $0.87 ; \mathrm{P}<0.001$. Risk of hospitalization for HF was $21 \%(\mathrm{P}<0.001)$ less in SAC/VAL group than in enalapril group (HR $0.79 ; 95 \% \mathrm{CI}, 0.71$ to 0.89 ). Additionally, SAC/VAL also significantly decreased the symptoms and physical limitations of heart failure $(P=0.001)$ as per the Kansas City Cardiomyopathy Questionnaire (KCCQ) clinical score [12]. Though, adverse events like renal impairment, hyperkalemia, and cough were lower in SAC/VAL group, yet hypotension and nonserious angioedema (no patient had airway compromise or required mechanical airway protection) were reported higher in SAC/VAL group compared to enalapril group [12].

\section{Current Treatment Guidelines for Using Sacubitril/ Valsartan Combination}

After the remarkable success of the PARADIGM-HF trial, SAC/VAL was approved in Europe and the United States for the treatment of adults with chronic HFrEF (NYHA class II-IV), to reduce the risk of cardiovascular death and hospitalization [3]. The 2016 update the European Society of Cardiology (ESC) guidelines for the diagnosis and treatment of acute and chronic heart failure and the 2017 update of the American College of Cardiology (ACC)/American Heart Association Task Force (AHA)/ the Heart Failure Society of America (HFSA) guidelines for HF, and the Canadian Cardiovascular Society Heart Failure included SAC/VAL therapy for patients with HFrEF (Table 1) [6-8].

\begin{tabular}{|c|c|c|}
\hline Guideline & $\begin{array}{l}\text { Recommendations on angiotensin receptor/neprilysin inhibitor } \\
\text { (ARNI) Therapy }\end{array}$ & $\begin{array}{l}\text { Class of Evidence and Level of } \\
\text { Recommendation }\end{array}$ \\
\hline $\begin{array}{l}\text { The European Society of Cardiology } \\
\text { (ESC) guidelines, } 2016[7]\end{array}$ & $\begin{array}{l}\text { In patients with symptomatic (NYHA Class II-IV) HFrEF, Sacubitril/ } \\
\text { valsartan is recommended as a replacement for an ACE-I to further } \\
\text { reduce the risk of HF hospitalization and death in ambulatory patients } \\
\text { with HFrEF who remain symptomatic despite optimal treatment with } \\
\text { an ACE-I, a beta-blocker and an MRA }\end{array}$ & $\begin{array}{l}\text { Level of Recommendation: Recommended/ } \\
\text { Indicated Class of Evidence: Single RCT }\end{array}$ \\
\hline $\begin{array}{l}\text { The American College of Cardiology } \\
\text { (ACC)/American Heart Association Task } \\
\text { Force (AHA)/ the Heart Failure Society of } \\
\text { America (HFSA) guidelines, } 2017 \text { [6] }\end{array}$ & $\begin{array}{l}\text { In patients with chronic symptomatic HFrEF NYHA class II or III who } \\
\text { tolerate an ACE inhibitor or ARB, replacement by an ARNI is } \\
\text { recommended to further reduce morbidity and mortality. } \\
\text { ARNI should not be administered to patients with a history of } \\
\text { angioedema. }\end{array}$ & $\begin{array}{l}\text { Level of Recommendation: Strong Class of } \\
\text { Evidence: Moderate Quality }\end{array}$ \\
\hline $\begin{array}{l}\text { The Canadian Cardiovascular Society } \\
\text { guidelines, } 2017 \text { [8] }\end{array}$ & $\begin{array}{l}\text { If patients with HFrEF remains symptomatic despite triple therapy, } \\
\text { consideration should be made to change an ACEi/ARB to an ARNI. to } \\
\text { decrease cardiovascular death, HF hospitalizations, and symptoms. } \\
\text { ARNIs should not be used in anyone with a history of angioedema. }\end{array}$ & $\begin{array}{l}\text { Level of Recommendation: Strong Class of } \\
\text { Evidence: High-Quality Evidence }\end{array}$ \\
\hline
\end{tabular}

Table 1: Current International Guidelines for using angiotensin receptor/neprilysin inhibitor (ARNI) therapy in patients with Heart failure.

However, a disparity is seen among the guidelines regarding use of SAC/VAL in NYHA class IV HF. The ESC and the Canadian guidelines recommend use of SAC/VAL in patients with class IV HF (albeit with only a modest representation of NYHA class IV in PARADIGM-HF trial) but, the AHA/ACC/HFSA guidelines limits the use of SAC/VAL only in patients with NYHA class II-III [6-8]. 


\section{Clinical Efficacy Sacubitril/Valsartan}

Following the PARADIGM-HF trial, SAC/VAL therapy became area of interest for HF researchers. Several trials were conducted to evaluate efficacy of SAC/VAL in patients with HF.

In a post-hoc analysis from PARADIGM-HF, a dose-reduction study was performed. This study compared the efficacy of SAC/VAL or enalapril if the patients were administered a dose lower than the target dose of respective drug [13]. During the trial, 3549 patients underwent to a dose reduction to $100 / 50 / 0 \mathrm{mg} \mathrm{SAC/VAL}(43 \%, \mathrm{n}=1755)$ or $5 / 2.5 / 0$ mg enalapril $(42 \%, n=1794)$ twice daily from the maximal dose of 200 $\mathrm{mg}$ SAC/VAL or $10 \mathrm{mg}$ enalapril twice daily [13]. The results of this study showed that efficacy of SAC/VAL over enalapril was similar among patients with dose reduction (HR 0.80, 95\% CI 0.70-0.93, $\mathrm{P}<0.001$ ) and those without dose reduction (HR 0.79, 95\% CI $0.71-$ $0.88, \mathrm{P}<0.001$ ). Hence, unlike enalapril the magnitude of benefits does not change if SAC/VAL was administered at lower dose [13]. The findings of these study are indicative of a consistent efficacy of $\mathrm{SAC/VAL}$ even in patients taking less than the target dose.

Early readmission after HF hospitalization worsen the prognosis and augments economic burden on the patient and policy makers. In patients with chronic HFrEF, SAC/VAL could reduce all-cause and heart-failure readmissions at 30 days during the therapy. In an analysis conducted to compare the rates of hospital readmission at 30-days following HF hospitalization of SAC/VAL with enalapril. The results of this study showed that the rates of all-cause and heart-failure readmissions at 30 days during treatment with SAC/VAL were fewer relative to enalapril $(9.7 \%$ vs. $13.4 \%$; odds ratio: 0.62 ; $95 \%$ CI: 0.45 to $0.87 ; \mathrm{P}=0.006)$ [14]. Hence, SAC/VAL could be more efficacious and cost-effective than enalapril in patients with HFrEF.

In a post-hoc exploratory analysis from PARADIGM-HF trial the effect of SAC/VAL or enalapril on systolic blood pressure (SBP) and on the primary composite outcome (cardiovascular death or heart failure hospitalization), its components and all-cause death was compared. The results of this analysis showed that benefit of SAC/VAL over enalapril was consistent across all baseline SBP categories for all outcomes [15]. The SAC/VAL versus enalapril hazard ratio for the primary endpoint was $0.88(95 \% \mathrm{CI} 0.74-1.06)$ in patients with a baseline SBP $<110 \mathrm{mmHg}$ and 0.81 (0.65-1.02) for those with an SBP $\geq$ $140 \mathrm{mmHg}$ ( $\mathrm{P}$ for interaction=0.55) [15]. Compared to other traditional therapies, SAC/VAL is beneficial across the range of SBP in HF patients. Patients with a higher SBP have shown better responsiveness than those with lower SBP with SAC/VAL. However, risk of hypotension-related adverse effects remains high in these patients.

Superior efficacy of SAC/VAL over enalapril irrespective of background therapy with diuretic, MRA, digoxin, and implanted cardiac defibrillator is also well documented. In a post hoc-analysis of PARADIGM-HF trial primary endpoints like cardiovascular death or heart failure hospitalization, as well as cardiovascular death were analyzed for both SAC/VAL and enalapril therapy. The findings from this study revealed that efficacy of SAC/VAL was consistent across all subgroups examined. The hazard ratio for primary end-point ranged from 0.74 to 0.85 and for cardiovascular death ranged from 0.75 to 0.89 , with no treatment-by-subgroup interaction [16]. Hence, unlike enalapril SAC/VAL can be easily used in HF patients regardless of background therapy and irrespective of previous coronary revascularization or $\beta$-blocker dose.
Another trial comparing SAC/VAL with valsartan reported that SAC/VAL was associated with short-term increases in natriuresis and diuresis, superior office and ambulatory blood pressure control, and significantly reduced $\mathrm{N}$-terminal pro B-type natriuretic peptide levels in Asian patients with salt-sensitive hypertension [17].

In a randomized, double-blind, placebo-controlled study SAC/VAL was compared to sacubitril (AHU377; a neprilysin inhibitor) for blood-pressure reduction in mild-to-moderate hypertensive patients. Unlike sacubitril (200 mg) alone, SAC/VAL (400 mg) showed full additivity for the reduction of mean sitting diastolic blood pressure. The findings of this study were indicative of better efficacy SAC/VAL over sacubitril alone in treatment of hypertension and cardiovascular disease [18].

\section{Clinical Safety Sacubitril/Valsartan}

After PARADIGM-HF trial, safety and tolerability of SAC/VAL is also studied in several other clinical trials. Neprilysin is also one of many enzymes associated with clearing amyloid-peptides from the brain. Hence, the long-term effects of SAC/VAL on cognition of the patient was questionable. However, a post-hoc analysis of PARADIGM-HF study reported that during the trial no evidence were found to show that SAC/VAL increases dementia-related side effects. Hence, SAC/VAL was similar in safety with enalapril in terms of adverse cognitive effects [19].

In an up-titration analysis from PARADIGM-HF trial, the tolerability of initiating/up titrating SAC/VAL from 50 to $200 \mathrm{mg}$ twice daily (target dose) over 3 and 6 weeks in heart failure (HF) patients $(\mathrm{n}=540$, ejection fraction $\leq 35 \%)$ was evaluated. The results of this study showed that both initiation and up titration regimens for SAC/VAL had a tolerability profile considered in line with other treatments for HF [20].

Likewise, single therapeutic and supratherapeutic doses of SAC/VAL were found to be safe and had no effect on cardiac repolarization as defined by the E14 International Conference on Harmonization (ICH) guidelines [21]. Another study reported that SAC/VAL did not cause changes in cerebrospinal fluid (CSF) levels of aggregable $A \beta$ isoforms (1-42 and 1-40) compared with placebo, despite achieving CSF concentrations of SAC/VAL sufficient for therapeutic activity [22].

\section{Effect of Sacubitril/Valsartan on Quality of Life (QoL)}

Health-related quality of life (HRQL) of patients from PARADIGMHF trial showed promising HRQL in surviving patients with heart failure on SAC/VAL therapy [23]. At 8 months, SAC/VAL group noted improvements in both KCCQ clinical summary score $(n=6881,+0.64$ versus $-0.29 ; \mathrm{P}=0.008)$ and KCCQ overall summary score $(\mathrm{n}=7623$, +1.13 versus $-0.14 ; \mathrm{P}<0.001$ ) in comparison to enalapril group and significantly less proportion of patients with deterioration ( $\geq 5$ points decrease) of both KCCQ scores (27\% versus $31 \% ; \mathrm{P}=0.01)$ [23].

In another secondary analysis $(\mathrm{n}=7618)$ of the PARADIGM-HF trial it was reported that SAC/VAL significantly improved nearly all KCCQ physical and social activities compared to enalapril. Improvement was seen in household chores and sexual relationships [24]. 


\section{Cost Effectiveness of Sacubitril/Valsartan}

For nearly two decades ACEIs, ARBs, and BBs have remained the foundation therapy for management of HFrEF [5]. However, in 2015, the PARADIGM HF trial demonstrated an exceptional safety and efficacy profile of ARNI therapy using SAC/VAL in management of HFrEF. Henceforth, it was recommended that SAC/VAL can be used to replace the ACEIs or ARB in HF patients with no history of angioedema. However, SAC/VAL should not be administered concomitantly with ACE inhibitors or within 36 hours of the last dose of an ACEIs [6-8].

Clinically, replacement of ACEIs or ARB with SAC/VAL in patients with HF have shown better efficacy, fewer adverse reactions, and improved quality of life (QoL). But, a higher drug acquisition cost of SAC/VAL compared to traditional therapies had made its clinical utility questionable. The cost-effectiveness of SAC/VAL is influenced by all-cause mortality benefit, SAC/VAL drug costs, probability of NYHA class progression, and assumed duration of treatment (i.e., model time horizon) [25].

In a cost-effectiveness study it was stated that though SAC/VAL reduces HF hospitalizations, but at the current price it is unlikely to be cost-effective. The wholesale acquisition cost of SAC/VAL ( $\$ 12.50$ per day) is much higher than popular ACEIs ( 0.13 per day) and is expected to fluctuate between different health care systems [26]. Furthermore, the cost-effectiveness of SAC/VAL was found to be dependent to the willingness to pay (WTP) threshold. The SAC/VAL therapy was likely to be cost-effective given a WTP of $\$ 100,000$ per quality-adjusted lifeyears (QALYs) or higher, but not at a WTP of $\$ 50,000$ per QALYs. The study also stated that if a generic version of SAC/VAL is made available in near future, the cost will drop from $10 \%$ to $50 \%$ of the current cost of the brand-name medication and the incremental cost-effectiveness ratio (ICER) will drop below $\$ 50,000$ per QALYs [26].

A pharmacoeconomic study compared the efficacy and costeffectiveness of SAC/VAL and enalapril in United States (US) population [27]. The findings of the study revealed that an average monthly cost for SAC/VAL was considerably higher (\$375) compared to enalapril (\$0.96). However, patients on SAC/VAL therapy showed a smaller number of hospitalizations compared to enalapril and higher QALYs, costs, and incremental costs per QALY gained [27]. It was reported that for every 100,000 people on SAC/VAL therapy, the overall medical savings from reduced HF hospitalization would be more than \$27 million in a year. Hence, despite a higher monthly drug accusation cost, SAC/VAL was found to be more cost-effective than enalapril as long-term therapy in patients with HF [27].

In a clinical study, a base-case analysis for the UK population indicated that SAC/VAL was cost-effective at a WTP threshold of \$ 25476 per QALY, compared with an ACEIs, with an incremental costeffectiveness ratio (ICER) of $\$ 21782$ [28]. Similar results were found for Denmark (SAC/VAL was cost-effective at WTP threshold of \$ 37913 per QALY, compared with an ACEI, with an ICER of \$26387) and Columbia (SAC/VAL was cost-effective at a WTP threshold of $\$ 16175$ per QALY, with an ICER of \$12131). Though, the results were promising for SAC/VAL, but it is worth mentioning that all three participating countries (UK, Denmark, and Columbia) in this trial were high to middle-income countries. Hence, cost-effectiveness of SAC/VAL remains uncertain in low-income countries [28].

A US based cost-effectiveness study reported SAC/VAL as only a marginally cost-effective treatment option. The SAC/VAL therapy has been cost-effective primarily at WTP thresholds of $\$ 150,000 /$ QALY gained or greater. However, other classes of drugs like ACEI, ARBs, and BBs were generally highly cost-effective (ICER $<\$ 10,000 /$ QALY gained) [25]. Likewise, cardiac resynchronization therapy and implantable cardioverter defibrillators were generally cost-effective with ICERs ranging from $\$ 20,000$ to $\$ 90,000 / \mathrm{QALY}$ gained. The findings of this study indicated the need for new pricing strategies to improve the cost-effectiveness of SAC/VAL [25].

Another important factor influencing the cost-effectiveness of SAC/VAL is that currently SAC/VAL is available only for HFrEF patients who are already on an ACEIs or ARB therapy [29]. A Dutch study showed that if a HF patient is already on ACEIs/ARB, adding SAC/VAL to the therapy would increase the health care cost by $\$ 2.69 \mathrm{a}$ day. However, a lower cost of SAC/VAL would help in bringing down the overall health budget [29]. The study also highlighted that in patients undergoing long-term therapy, unless SAC/VAL is priced lower than enalapril ( $<\$ 0.02$ per day) is very unlikely to be cost-saving compared to the standard therapy [29]. Similar issues were addressed in an Asian study conducted in Singapore. The findings reveled that at current daily price of $\$ 6.49$ SAC/VAL may not be cost-effective compared to enalapril in reducing cardiovascular morbidity and mortality in HF in Singapore healthcare setting [30].

These cost-effective analyses suggest SAC/VAL as a cost-effective resource. Though SAC/VAL is an expensive than the traditional drugs for patients with HFrEF, but better efficacy, comparably less adverse events, and reduced number of hospitalizations makes it cost-effective than other drugs in long term therapy.

\section{Upcoming Clinical Research}

Findings from epidemiological studies indicated that $50 \%$ of patients with heart failure have HFpEF and the proportion of HFpEF patients is increasing steadily. Worrisome is the fact that no conventional heart failure therapy has shown to improve patient outcomes in HFpEF patients. Hence, making this group of patients ideal to reconnoiter the potential of new SAC/VAL therapy [31].

The upcoming prospective comparison of ARNI with ARB global outcomes in heart failure with preserved ejection fraction (PARAGON-HF) trial is set to explore role of SAC/VAL in HFpEF. The primary endpoint of this trial is to compare SAC/VAL to valsartan in reducing the rate of the cardiovascular death and total (first and recurrent) HF hospitalizations, in HFpEF patients (NYHA Class II-IV; LVEF $\geq 45 \%$ ). Findings of this trial will further explore the clinical usage of SAC/VAL in this sub group of HF patients [32].

The PARALLAX trial (NCT03066804) is another ongoing 24-week, a randomized, double-blind controlled study to evaluate the effect of SAC/VAL on NT-proBNP, symptoms, exercise function, and safety compared to individualized medical management of comorbidities in patients with HFpEF.

The mechanism of action of SAC/VAL and previous experimental and clinical data seems to suggest its possible success in HFpEF. However, the controversial multifactorial pathophysiologic mechanisms involved in HFpEF makes it challenging to elucidate the efficacy of SAC/VAL therapy in these patients. Hence, further testing of SAC/VAL in HFpEF is necessary to elucidate its safety and efficacy profile in this population.

Two other important clinical trials are scheduled to explore the therapeutic use of SAC/VAL in HF. The PANORAMA-HF study, a prospective pediatric HF trial is to be conducted using a global rank 
primary endpoint. The findings of this study will determine whether SAC/VAL is superior to enalapril for treatment of pediatric HF patients with reduced systemic left ventricular systolic function $[33,34]$.

Another study namely, the ENTRESTO-SAS trial will be a 3-month, multicentric, prospective, open-label real-life cohort study. This study will evaluate whether SAC/VAL could improve the outcome of sleepdisordered breathing in chronic HF patients [32]. Findings of these trials would further expand the clinical use of SAC/VAL and a diverse patient group is likely to be benefited from this drug in near future.

\section{Conclusion}

Introduction of SAC/VAL revolutionized the management of HF. The findings of the initial trial proved the superiority of SAC/VAL over enalapril in patients with HFrEF. Though, the safety and efficacy of SAC/VAL is proven in patients with HF, yet the therapeutic role of this combination is yet to be elucidated in HFpEF and other special population groups. The cost-effective studies for SAC/VAL in HF indicate it to be an expensive therapy than traditional drugs available for management of HF. It is worth mentioning that despite being expensive SAC/VAL exhibits a better safety and efficacy profile than most of the conventional drug. When considered for long-term therapy SAC/VAL shows better tolerability, is equally efficacious at lower the target dose, reduces incidence of possible adverse events, shows less severe signs of angioedema, improves overall QoL, and reduce the chances of early rehospitalization with ongoing therapy. Therefore, indirectly SAC/VAL reduces the overall cost of health care with ongoing HF therapy as compared to conventional therapies.

However, the cost-effectiveness of the SAC/VAL is still questionable in developing regions of the world. Credible data is not available to demonstrate the proportion of usage of SAC/VAL in developing countries. Currently, SAC/VAL is priced much above the other standard-of-care in HF and has proven to be a cost-effective only in high-medium income countries where most of the patients are benefited by medical insurance and federal healthcare policies. Till date no pharmacoeconomic studies are done to evaluate the costeffectiveness of SAC/VAL in developing countries to elucidate its role in these regions of the world. Furthermore, in real-world clinical practice, it is difficult for a physician to convince a patient from poor economic background to select SAC/VAL which at first instance is much costlier than conventional drugs.

Hence, robust long-term, real-world estimates of effectiveness are needed to fully understand the cost-effectiveness of SAC/VAL therapy. It is worth mentioning that due to high costs of SAC/VAL, initiation of therapy requires a judicious decision among all the healthcare stakeholders. Therefore, measures like lower pricing, and better federal healthcare policies (in developing countries) are required to overcome these limitations and to ensure maximum patients are benefited by SAC/VAL.

\section{References}

1. Ambrosy PA, Fonarow GC, Butler J (2014) The Global Health and Economic Burden of Hospitalizations for Heart Failure. Lessons Learned from Hospitalized Heart Failure Registries. J Am Coll Cardiol 63: 1123-1133.

2. Kemp CD, Conte JV (2012) The pathophysiology of heart failure. Cardiovasc Pathol 21: 365-371.
3. Liu RC (2018) Focused Treatment of Heart Failure with Reduced Ejection Fraction Using Sacubitril/Valsartan. Am J Cardiovasc Drugs.

4. Dokainish H, Teo K, Zhu J (2016) Heart failure in Africa, Asia, the Middle East and South America: The INTER-CHF study. Int J Cardiol 204: 133-141.

5. Redfield MM (2016) Heart Failure with preserved ejection fraction. N Engl J Med 75: 1868-1877.

6. Yancy CW, Jessup M, Bozkurt B (2016) ACC/AHA/HFSA Focused Update on New Pharmacological Therapy for Heart Failure: An Update of the 2013 ACCF/AHA Guideline for the Management of Heart Failure: A Report of the American College of Cardiology/American Heart Association Task Force on Clinical Practice Guidelines and the Heart Failure Society of America. J Am Coll Cardiol 68: 1476-1488.

7. Ponikowski P, Voors AA, Anker SD (2016) ESC Guidelines for the diagnosis and treatment of acute and chronic heart failure: The Task Force for the diagnosis and treatment of acute and chronic heart failure of the European Society of Cardiology (ESC)Developed with the special contribution of the Heart Failure Association (HFA) of the ESC. Eur Heart J 37: 2129-2200.

8. Ezekowitz JA, O'Meara E, McDonald MA (2017) Comprehensive Update of the Canadian Cardiovascular Society Guidelines for the Management of Heart Failure. Can J Cardiol 33: 1342-1433.

9. Mozaffarian D, Benjamin EJ, Go AS (2016) Heart disease and stroke statistics-2016 update: a report from the American Heart Association. Circulation 133: e38-360.

10. Bayés GA (2015) Neprilysin in heart failure: from oblivion to center stage. JACC Heart Fail 3: 637-640.

11. Egea OI, Montón GC, Roura S (2017) Mechanisms of action of sacubitril/ valsartan on cardiac remodeling: a systems biology approach. NPJ Syst Biol Appl 3: 12.

12. McMurray JJ, Packer M, Desai AS (2014) Angiotensin-neprilysin inhibition versus enalapril in heart failure. N Engl J Med 37: 993-1004.

13. Vardeny O, Claggett B, Packer M (2016) Efficacy of sacubitril/valsartan vs. enalapril at lower than target doses in heart failure with reduced ejection fraction: the PARADIGM-HF trial. Eur J Heart Fail 18: 1228-1234.

14. Desai AS, Claggett BL, Packer M (2016) Influence of Sacubitril/Valsartan (LCZ696) on 30-Day Readmission After Heart Failure Hospitalization. J Am Coll Cardiol 68: 241-248.

15. Böhm M, Young R, Jhund PS (2017) Systolic blood pressure, cardiovascular outcomes and efficacy and safety of sacubitril/valsartan (LCZ696) in patients with chronic heart failure and reduced ejection fraction: results from PARADIGM-HF. Eur Heart J 38: 1132-1143.

16. Okumura N, Jhund PS, Gong J (2016) Effects of Sacubitril/Valsartan in the PARADIGM-HF Trial (Prospective Comparison of ARNI with ACEI to Determine Impact on Global Mortality and Morbidity in Heart Failure) According to Background Therapy. Circ Heart Fail P: 9.

17. Wang TD, Tan RS, Lee HY (2017) Effects of Sacubitril/Valsartan (LCZ696) on Natriuresis, Diuresis, Blood Pressures, and NT-proBNP in Salt-Sensitive Hypertension. Hypertension 69: 32-41.

18. Ruilope LM, Dukat A, Böhm M (2010) Blood-pressure reduction with LCZ696, a novel dual-acting inhibitor of the angiotensin II receptor and neprilysin: a randomised, double-blind, placebo-controlled, active comparator study. Lancet 375:1255-1266.

19. Cannon JA, Shen L, Jhund PS (2017) Dementia-related adverse events in PARADIGM-HF and other trials in heart failure with reduced ejection fraction. Eur J Heart Fail 19:129-137.

20. Senni M, McMurray JJ, Wachter R (2016) Initiating sacubitril/valsartan (LCZ696) in heart failure: results of TITRATION, a double-blind, randomized comparison of two uptitration regimens. Eur J Heart Fail 18: 1193-1202.

21. Langenickel TH, Jordaan P, Petruck J (2016) Single therapeutic and supratherapeutic doses of sacubitril/valsartan (LCZ696) do not affect cardiac repolarization. Eur J Clin Pharmacol 72: 917-24. 
Citation: Ellahham S (2018) Sacubitril/Valsartan in Management of Heart Failure: A Review of Safety, Efficacy and Cost-Effectiveness. Cardiovasc Pharm Open Access 7: 250. doi:10.4172/2329-6607.1000250

Page 7 of 7

22. Langenickel TH, Tsubouchi C, Ayalasomayajula S (2016) The effect of LCZ696 (sacubitril/valsartan) on amyloid- $\beta$ concentrations in cerebrospinal fluid in healthy subjects. Br J Clin Pharmacol 81: 878-890.

23. Lewis EF, Claggett BL, McMurray JJV (2017) Health-Related Quality of Life Outcomes in PARADIGM-HF. Circ Heart Fail 10.

24. Chandra A, Lewis EF, Claggett BL, Desai AS (2018) Effects of Sacubitril/ Valsartan on Physical and Social Activity Limitations in Patients with Heart Failure A Secondary Analysis of the PARADIGM-HF Trial. JAMA Cardiol 3:498-505.

25. Zueger PM, Kumar VM, Harrington RL (2018) Cost-Effectiveness Analysis of Sacubitril/Valsartan for the Treatment of Heart Failure with Reduced Ejection Fraction in the United States. Pharmacotherapy 38: 520-530.

26. King JB, Shah RU, Bress AP (2016) Cost-Effectiveness of SacubitrilValsartan Combination Therapy Compared with Enalapril for the Treatment of Heart Failure With Reduced Ejection Fraction. JACC Heart Fail 4:392-402.

27. Gaziano TA, Fonarow GC, Claggett B (2016) Cost-effectiveness Analysis of Sacubitril/Valsartan vs Enalapril in Patients with Heart Failure and Reduced Ejection Fraction. JAMA Cardiol 1:666-672.

28. McMurray JJV, Trueman D, Hancock E (2018) Cost-effectiveness of sacubitril/valsartan in the treatment of heart failure with reduced ejection fraction Heart 104:1006-1013.
29. van der Pol S, Degener F, Postma MJ (2017) An Economic Evaluation of Sacubitril/Valsartan for Heart Failure Patients in the Netherlands. Value Health 20: 388-396.

30. Liang L, Bin-Chia Wu D, Aziz MIA (2018) Cost-effectiveness of sacubitril/valsartan versus enalapril in patients with heart failure and reduced ejection fraction. J Med Econ 21:174-181.

31. Redfield MM (2016) Heart Failure with preserved ejection fraction. N Engl J Med 375:1868-1877.

32. Solomon SD, McMurray JJ, Gong J (2018) Long-term efficacy and safety comparison of the angiotensin receptor neprilysin inhibitor (ARNi), LCZ696, and valsartan, in patients with heart failure and preserved ejection fraction: A randomized, double blind, morbidity and mortality trial Prospective comparison of ARni with Arb Global Outcomes in heart failure with preserved ejectioN fraction (PARAGON-HF). Circ Heart Fail 11: e004962.

33. Shaddy R, Canter C, Halnon N (2017) Design for the sacubitril/valsartan (LCZ696) compared with enalapril study of pediatric patients with heart failure due to systemic left ventricle systolic dysfunction (PANORAMAHF study). Am Heart J 193:23-34.

34. Jaffuel D, Molinari N, Berdague P (2018) Impact of sacubitril-valsartan combination in patients with chronic heart failure and sleep apnoea syndrome: the ENTRESTO-SAS study design. ESC Heart Fail 5:222. 